\title{
Feasibility of laparoscopic gastrectomy for patients with poor physical status: a retrospective cohort study based on a nationwide registry database in Japan
}

\author{
Mikito Inokuchi ${ }^{1}$ (1) Hiraku Kumamaru² Masatoshi Nakagawa $^{3} \cdot$ Hiroaki Miyata $^{2} \cdot$ Yoshihiro Kakeji $^{4} \cdot$ \\ Yasuyuki Seto ${ }^{5} \cdot$ Kazuyuki Kojima ${ }^{6}$
}

Received: 26 March 2019 / Accepted: 15 July 2019 / Published online: 22 July 2019

(c) The International Gastric Cancer Association and The Japanese Gastric Cancer Association 2019

\begin{abstract}
Background Laparoscopic gastrectomy (LG) is an established minimally invasive procedure for gastric cancer. However, it is controversial whether LG is useful for patients with poor physical status classified into higher classes of the American Society of Anesthesiologists physical status (ASA-PS) classification. The aim of this study was to determine the feasibility of LG in patients with ASA-PS class $\geq 3$.

Methods We extracted data for a total of 28,160 patients with an ASA-PS class $\geq 3$ who underwent distal or total gastrectomy for gastric cancer between January 2013 and December 2017 from the National Clinical Database Japan society for gastroenterological surgery registry. We developed a propensity score model from baseline demographics and comorbidities and matched patients undergoing LG to those undergoing open gastrectomy (OG) using a 1:1 ratio. Mortality and morbidities (within 30 days and in-hospital) were compared between the 6998 matched patient pairs.

Results In-hospital mortality was significantly lower in patients undergoing LG than in those undergoing OG (2.3\% vs. $3.0 \%$, $p=0.01$ ), while the 30 -day mortality was similar (1.6\% vs. $1.5 \%)$. The length of hospital stay was significantly shorter in the LG group (median, 14 days vs. 17 days, $p<0.001$ ). The LG group had a significantly lower incidence of postoperative complications in patients with any grade complication $(20.3 \%$ vs. $22.5 \%, p=0.002)$ as well as those with $\geq$ grade 3 complications $(8.7 \%$ vs. $9.8 \%, p=0.03)$.

Conclusion LG was associated with decreased in-hospital mortality and a lower incidence of several postoperative complications when compared to OG among patients with poor physical condition.
\end{abstract}

Keywords Gastric cancer · Laparoscopic gastrectomy · Open gastrectomy · American society of anesthesiologists' performance status $\cdot$ Postoperative complications

Mikito Inokuchi

m-inokuchi.srg2@tmd.ac.jp

1 Department of Surgery, Japanese Red Cross Musashino Hospital, 1-26-1 Kyonan-cho, Musashino 180-8610, Tokyo, Japan

2 Department of Health Quality Assessment, Graduate School of Medicine, University of Tokyo, Tokyo, Japan

3 Department of Gastrointestinal Surgery, Graduate School of Medicine, Tokyo Medical and Dental University, Tokyo, Japan

4 Database Committee, The Japanese Society of Gastroenterological Surgery, Tokyo, Japan

5 The Japanese Society of Gastroenterological Surgery, Tokyo, Japan

6 First Department of Surgery, Dokkyo Medical University, Mibu, Tochigi, Japan

\section{Introduction}

Laparoscopic gastrectomy (LG) has been established as a minimally invasive procedure for early-stage gastric cancer, with this procedure being acknowledged as the standard treatment option for clinical stage I gastric cancer in the Japanese guidelines [1]. Compared to open distal gastrectomy (ODG), laparoscopic distal gastrectomy (LDG) has shown several benefits such as lower amounts of bleeding, less pain, faster recovery, and shorter hospital stays in several Asian randomized controlled trials (RCTs) [2-4]. Moreover, postoperative complications were not significantly different between patients undergoing LDG or ODG, except for wound problems [4]. 
Laparoscopic surgery is considered contraindicated for patients having poor physical status such as cardiac, pulmonary, or renal dysfunction; because of this, most of these patients are commonly excluded from prospective surgical studies. During laparoscopic surgery, peritoneal insufflation of carbon dioxide $\left(\mathrm{CO}_{2}\right)$ induces several hemodynamic changes: increased mean arterial pressure, systemic and pulmonary vascular resistance, and reduced cardiac output [5]. In addition, increased $\mathrm{CO}_{2}$ and peritoneal pressure adversely affected respiratory function by causing hypercarbia and reduced pulmonary ventilation [6]. It is controversial whether LG is useful for patients with poor physical status, although several single-institutional studies have supported the feasibility of LG in patients with pulmonary disease, heart disease, or poor physical status as compared with open gastrectomy (OG) [7-9].

The American Society of Anesthesiologists physical status (ASA-PS) is a 6-category classification that has generally been used to conduct preoperative risk stratification regarding comorbidities or the physical condition of patients [10]. The anesthesiologist-assessed ASA-PS scale is considered as a simple and reliable method in clinical practice [11]. A greater ASA-PS class was associated with increased postoperative mortality or morbidity in various types of surgery [12-14]. In distal and total gastrectomy, including both open and laparoscopic approaches, ASA-PS class 3 or higher was shown to be an independent risk factor of postoperative mortality and morbidity [15-17]. In a multicenter study, there was $84 \%$ agreement between responsible anesthesiologists and other blinded anesthesiologists who had reviewed a duplicate version of the same medical record with respect to patients assigned to ASA-PS $>3$ [18].

The feasibility of LG has not been revealed in patients with ASA-PS class $\geq 3$ and dysfunction of a primary organ. Therefore, the aim of this study was to determine the feasibility of LG as compared with that of OG in patients with ASA-PS class $\geq 3$.

\section{Material and methods}

\section{Data source}

We utilized data from the Japan Society of Gastroenterological Surgery (JSGS) registry housed in the National Clinical Database (NCD), which is a nationwide web-based data entry system for surgical procedures in Japan [19]. The NCD was selected as the data source to reflect the current status of major surgeries in Japan, because it includes data from both high-volume or specialized centers to medium-sized or small general hospitals. Moreover, the NCD is linked to the surgical board certification system in the Japan Data registration and is mandatory for participating hospitals that seek hospital or surgeon certification from the society. The database is one of the largest-scale surgical databases and covers more than $90 \%$ of the general surgeries performed in Japan $[20,21]$. The data accuracy of the NCD was assessed using 45 items from the cases registered, in comparison with the medical records. The accuracy with the maximum postoperative variables was $>95 \%$, and that of mortality and status 30 days after the surgery was $>99 \%$ [22].

\section{Patient cohort}

We retrieved data for patients who underwent total gastrectomy or distal gastrectomy for gastric cancer between January 2013 and December 2017 from the NCD JSGS registry. We excluded patients who underwent surgery for the coresection of organs other than the gallbladder or spleen and those who had missing data regarding gender, age, or 30-day mortality. We also excluded patients with a recorded ASAPS class of 1 or 2. Using laparoscopy information, patients were divided into LG and OG groups.

\section{Study outcomes}

The primary outcome of interest was perioperative mortality. We assessed the incidence of death within 30 days of the procedure, as well as that of in-hospital mortality. Secondary outcomes included the incidence of postoperative complications, reoperation, and readmission within 30 days post-surgery, operating time and blood loss. Complications were classified using the Clavien-Dindo classification and we assessed the incidence of those with grade 3 and above [23]. Selected complications included leakage, pancreatic fistula (grades B and C according to the International study group of postoperative pancreatic fistula criteria), ileus, major bleeding (intraabdominal or intraluminal), wound dehiscence, surgical site infection, abscess, pneumonia, urinary tract infection, sepsis, cardiac event, cerebrovascular accident, acute hepatic failure, acute renal failure, and venous thromboembolism.

\section{Confounding factors}

With reference to previous studies from the NCD [24, 25], we considered the following patient-level and operative factors as potential confounders in the analysis: patient age, gender; ASA-PS class; body mass index; preoperative conditions including body weight loss greater than $10 \%$ within the past 6 months; smoking status; presence of habitual alcohol intake; activities of daily living; presence of comorbidities such as insulin-dependent diabetes mellitus, respiratory disease, chronic obstructive pulmonary disease, hypertension, and angina; hemodialysis; congestive heart failure; history of cerebrovascular accident; long-term use of steroids; and 
bleeding disorders. The type of gastrectomy (total or distal gastrectomy), presence of concurrent surgery (cholecystectomy and splenectomy), emergent surgery, use of preoperative chemotherapy, and tumor status based on the TNM classification were also considered as potential confounding variables.

\section{Propensity score matching and statistical analysis}

We tabulated patient characteristics for the LG and OG groups using counts and percentages for categorical variables. The differences in percentages between the two groups were assessed using the standard mean difference (SMD), as calculated using the publicly available macro by Yang and Dalton [26]. We then modeled the probability of undergoing LG according to all of the potential confounding factors using logistic regression, and used the predicted probability (propensity score: PS) for matching at a ratio of $1: 1$. The matching was conducted using a greedy matching algorithm without replacement, which was created by Marcelo Coca-Perraillon [27], and used a caliper of 0.2 standard deviation of $\log$ (PS). The analyst (H.K.) conducted the propensity score modeling and matching while being blinded to patient outcomes. The balance of the matched cohort was again assessed using a SMD below 0.1 as a good balance between the two groups. We then compared the incidences of the above-listed outcomes between the matched groups using Fisher's exact test for the outcomes with an expected cell count of less than 5, or a Pearson's Chi-squared test for other variables. Differences in operation time, operative blood loss, and length of postoperative stay were compared using the Wilcoxon rank-sum test. Comparisons were all two-sided and $p$ values less than 0.05 were considered statistically significant. All analyses were conducted using SAS 9.4 (SAS Institute, Cary, NC, USA).

\section{Results}

\section{Patient selection}

The flow chart for patient selection and analysis in this study is shown in Fig. 1. A total of 264,160 patients who underwent distal gastrectomy (including pylorus-preserving distal gastrectomy) or total gastrectomy (including proximal gastrectomy) had been registered in the NCD between 2013 and 2017. Of these, we excluded 39 patients who lacked essential data components, 8036 patients who did not undergo surgery to treat gastric cancer, and 12,787 patients who underwent co-resection of organs other than the gallbladder and spleen, and 215,138 patients with ASA-PS class 1 or 2 . The remaining 28,160 patients with ASA-PS class $\geq 3$ were included in the final analysis. Among them, 19,995 (71.0\%) had undergone OG and 8165 (29.0\%) underwent LG. Cases of conversion from OG to LG were registered as LG in the NCD starting in 2014. Conversion cases in 2013 may have been registered as OG, as there was no clear policy for registration during that time period; however, we estimated that it would have influenced less than $0.2 \%$ of the overall OG cohort, considering the conversion rate of $4.2 \%$ assessed using data from 2016-17.

\section{Patient characteristics before and after propensity score matching}

Background characteristics are shown in Table 1. Patients who were treated with OG tended to have more advanced
Fig. 1 Flow chart of patient selection and analysis in this study

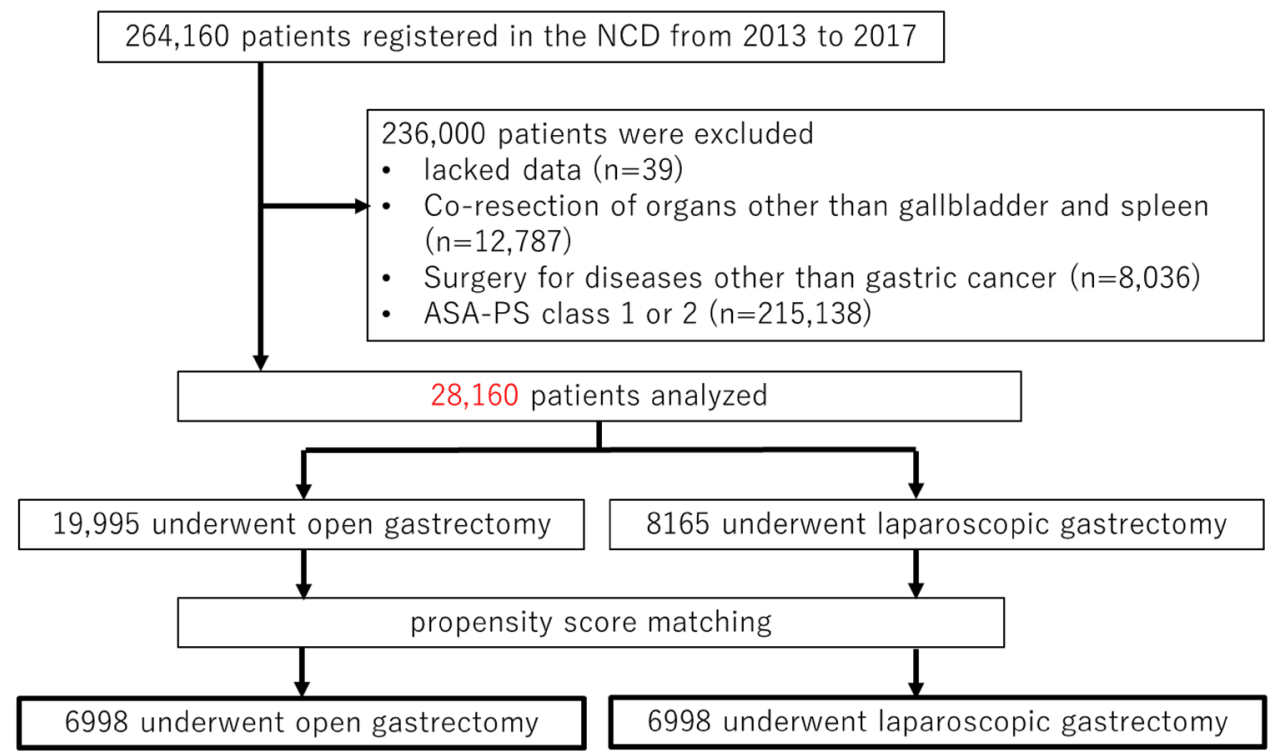




\begin{tabular}{|c|c|c|c|c|c|c|c|}
\hline & के & $\overline{0}$ & $\overline{0}$ & $\begin{array}{c}\overrightarrow{0} \\
i \\
1\end{array}$ & $\stackrel{\circ}{\circ}$ & 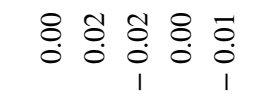 & 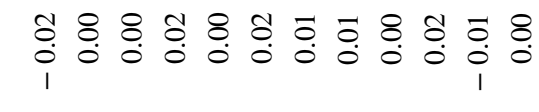 \\
\hline & $\therefore$ & $\stackrel{\infty}{+} \stackrel{\circ}{g} \stackrel{n}{g} \stackrel{0}{\dot{q}}$ & 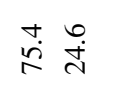 & $n_{n}^{n}=$ & 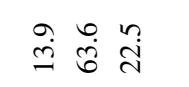 & 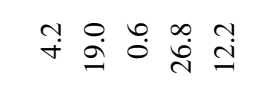 & 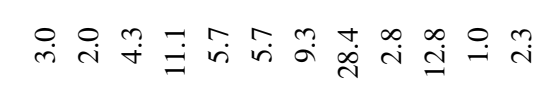 \\
\hline 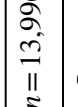 & 总育 & 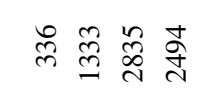 & 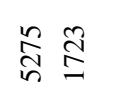 & $\stackrel{+a t}{\stackrel{t}{0}}$ & 㔯表点 & 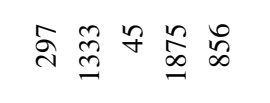 & 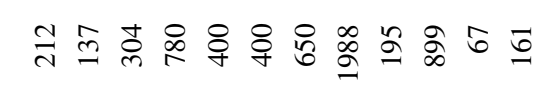 \\
\hline 离 & $\therefore$ & ?े & :ُ & $\stackrel{m}{\infty}$ & 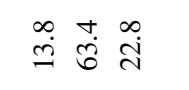 & $\stackrel{m}{m} \stackrel{m}{\infty} \stackrel{\infty}{\stackrel{0}{\infty}} \stackrel{0}{\stackrel{i}{i}} \stackrel{0}{i}$ & 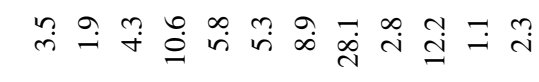 \\
\hline 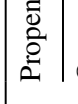 & 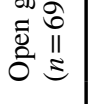 & 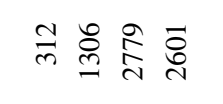 & 学点 & 略 & 旁等哭 & 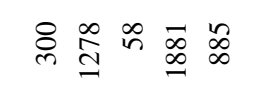 & 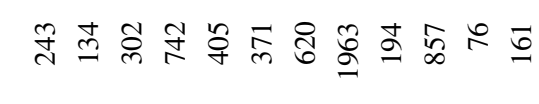 \\
\hline & ही & $\stackrel{9}{\circ}$ & $\begin{array}{l}\overline{0} \\
i \\
i\end{array}$ & $\stackrel{8}{0}$ & $\overline{9}$ & 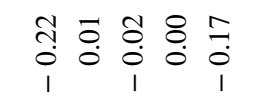 & 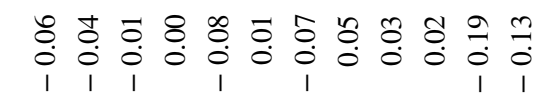 \\
\hline & so & 藏兽官 & 苦藏 & $\stackrel{\leftrightarrow}{\infty} \stackrel{m}{a}$ & 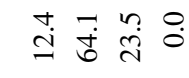 & 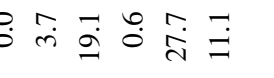 & 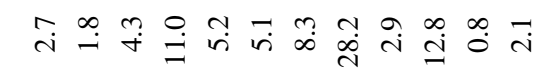 \\
\hline & 产育 & 㠻学命 & 总 㔛 & 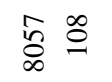 & 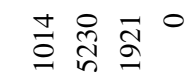 & 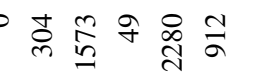 & 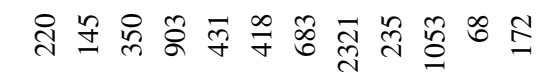 \\
\hline ô & so & 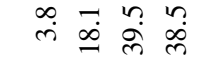 & 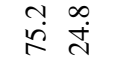 & 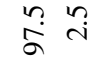 & 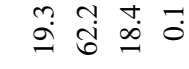 & 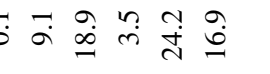 & 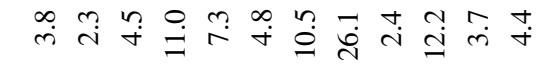 \\
\hline & $\begin{array}{ll} & \\
0 & \end{array}$ & 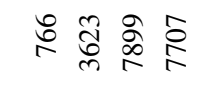 & 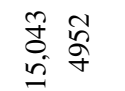 & 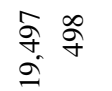 & 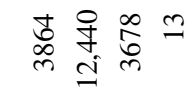 & 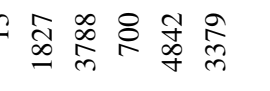 & ઈั \\
\hline & & 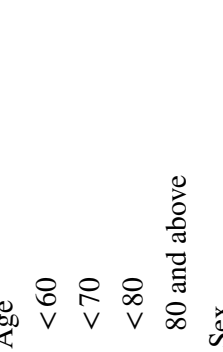 & & & 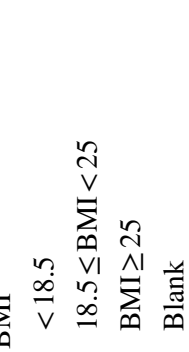 & 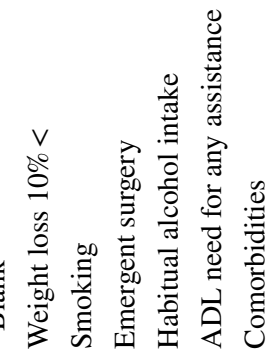 & 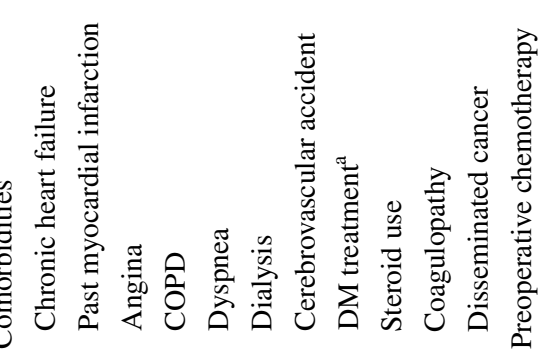 \\
\hline
\end{tabular}




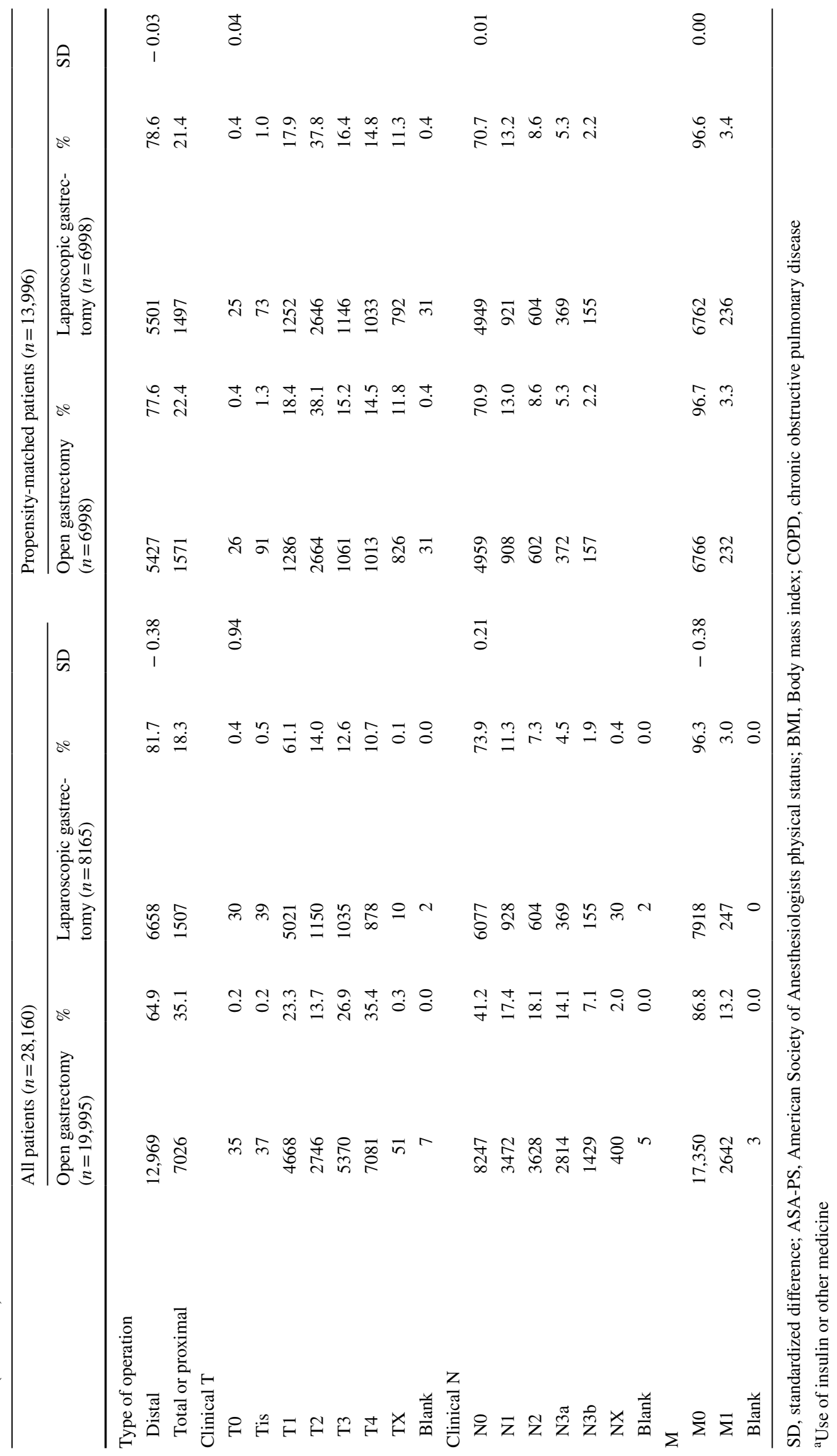


tumors ( $76.7 \%$ vs. $38.9 \%$ ), lymphatic metastasis (58.8\% vs. $26.1 \%)$, and distant metastatic disease (13.2\% vs. $3.0 \%)$ with preoperative body weight loss $(9.1 \%$ vs. $3.7 \%)$ or poor activities of daily living (16.9\% vs. $11.1 \%$ ). Total gastrectomy or proximal gastrectomy was more frequent in the OG group (35.1\% vs. $18.3 \%$ ). Almost all patents were classified as ASA-PS class 3 in both the OG (97.5\%) and LG (98.5\%) groups. After propensity score matching, by which information from 6998 patients who underwent OG and 6998 who underwent LG was retrieved, the standardized difference of all these confounding factors was reduced to 0.04 or less (Table 1). The bias of patient characteristics between the two groups was minimized considerably by propensity score matching; most differences were found in the clinical T stage.

\section{Comparison of morbidity and mortality between OG and LG}

Patient outcomes based on gastrectomy type are shown in Table 2. Operating time was longer for the LG group (median time: 300 min vs. $224 \mathrm{~min}, p<0.001$ ), whereas blood loss was lower for this treatment type (median amount: $50 \mathrm{~mL}$ vs. $230 \mathrm{~mL}, p<0.001)$. In-hospital mortality was lower in the LG group ( $2.3 \%$ vs. $3.0 \%, p=0.01)$, although there was no statistically significant difference in 30-day mortality. The length of postoperative hospital stay was shorter in the LG group (median time: 14 days vs. 17 days, $p<0.001$ ), although both the incidence of readmission and reoperation within 30 days after surgery did not differ between the two treatment groups.
Table 2 Short-term surgical outcomes after gastrectomy

\begin{tabular}{|c|c|c|c|c|c|}
\hline & $\begin{array}{l}\text { Open gastrec- } \\
\text { tomy }(n=6998)\end{array}$ & $(\%)$ & $\begin{array}{l}\text { Laparoscopic gas- } \\
\text { trectomy }(n=6998)\end{array}$ & $(\%)$ & $p$ value \\
\hline \multicolumn{6}{|l|}{ Operating time } \\
\hline Median (p10-p90) & $224(115-381)$ & & $300(175-485)$ & & $<0.001$ \\
\hline \multicolumn{6}{|l|}{ Blood loss } \\
\hline Median (p10-p90) & $230(26-1096)$ & & $50(0-570)$ & & $<0.001$ \\
\hline \multicolumn{6}{|l|}{ Length of postoperative stay } \\
\hline Median (p10-p90) & $17(9-72)$ & & $14(7-61)$ & & $<0.001$ \\
\hline \multicolumn{6}{|l|}{ Mortality } \\
\hline Within 30 days & 113 & 1.6 & 103 & 1.5 & 0.54 \\
\hline In-hospital & 208 & 3.0 & 159 & 2.3 & 0.01 \\
\hline Re-admission & 193 & 2.8 & 215 & 3.1 & 0.18 \\
\hline Re-operation within 30 days & 238 & 3.4 & 210 & 3.0 & 0.27 \\
\hline \multicolumn{6}{|l|}{ Complication $^{\mathrm{a}}$} \\
\hline Any complication & 1573 & 22.5 & 1424 & 20.3 & 0.002 \\
\hline Any complication of CD3 and above & 687 & 9.8 & 612 & 8.7 & 0.03 \\
\hline leakage & 245 & 3.5 & 280 & 4.0 & 0.12 \\
\hline Pancreatic fistula (grade B,C) & 129 & 1.8 & 128 & 1.8 & 0.95 \\
\hline Ileus & 142 & 2.0 & 100 & 1.4 & 0.007 \\
\hline Bleeding & 57 & 0.8 & 79 & 1.1 & 0.06 \\
\hline Wound dehiscence & 85 & 1.2 & 31 & 0.4 & $<0.001$ \\
\hline Superficial/deep SSI & 241 & 3.4 & 157 & 2.2 & $<0.001$ \\
\hline Abscess & 270 & 3.9 & 272 & 3.9 & 0.93 \\
\hline Pneumonia & 399 & 5.7 & 361 & 5.2 & 0.16 \\
\hline Urinary tract infection & 68 & 1.0 & 65 & 0.9 & 0.79 \\
\hline Sepsis & 143 & 2.0 & 121 & 1.7 & 0.17 \\
\hline Cardiac event & 97 & 1.4 & 66 & 0.9 & 0.02 \\
\hline Cerebrovascular disease & 44 & 0.6 & 37 & 0.5 & 0.44 \\
\hline Acute hepatic failure & 12 & 0.2 & 7 & 0.1 & 0.36 \\
\hline Acute renal failure & 109 & 1.6 & 105 & 1.5 & 0.78 \\
\hline PE/DVT & 28 & 0.4 & 31 & 0.4 & 0.70 \\
\hline
\end{tabular}

$p$ values derived from Wilcoxon rank-sum test for continuous variables and Pearson's Chi-squared or Fisher's exact test for binary variables

CD, Clavien-Dindo classification; SSI, surgical site infection; PE, pulmonary embolism; DVT, deep venous thrombosis

${ }^{\mathrm{a}}$ Individual complications are for those graded as CD 1 through 5 
The LG group had a lower incidence of postoperative complications, for any grade complication $(20.3 \%$ vs. $22.5 \%$, $p=0.002)$ as well as $\geq$ grade 3 complications ( $8.7 \%$ vs. $9.8 \%$, $\mathrm{p}=0.03)$. The LG group had a lower incidence of wound infection $(2.2 \%$ vs. $3.4 \%, p<0.001)$, dehiscence $(0.4 \%$ vs. $1.2 \%, p<0.001)$, ileus ( $1.4 \%$ vs. $2.0 \%, p=0.007)$, and cardiac events $(0.9 \%$ vs. $1.4 \%)$, while the incidence of bleeding complications tended to be lower in the OG group (1.1\% vs. $0.8 \%, p=0.06)$. There were no significant differences in the incidence of critical surgical complications such as leakage, intraabdominal abscess, and pancreatic fistula. Infectious diseases such as pneumonia, urinary tract infection, and sepsis were not significantly different between the LG and OG treatment groups (5.2\% vs. $5.7 \%, 0.9 \%$ vs. $1.0 \%$, and $1.7 \%$ vs. $2.0 \%$, respectively). In addition, other medical complications (e.g., cerebrovascular disease, acute hepatic failure, and acute renal failure) did not differ between the two groups.

\section{Discussion}

This study examined the feasibility of LG in patients with ASA-PS class $\geq 3$ and dysfunction of a primary organ, as compared to those who underwent OG. Our results suggest that LG is tolerable in risky patients with a poor physical status, as indicated by an ASA-PS class of 3 or 4 . Patients undergoing LG had fewer complications than those undergoing OG. Despite longer operating times, as well as adverse effects on the cardiopulmonary systems possibly caused by pneumoperitoneum, LG was not associated with increased postoperative cardiopulmonary or other types of medical complications in our study. Furthermore, both in-hospital mortality and the incidence of cardiac events were less frequent in patients undergoing $\mathrm{LG}$, when compared to those undergoing OG. The progression of postoperative comorbidities that existed prior to surgery might be lower for patients undergoing LG because the 30-day mortality rate and the incidence of critical surgical complications did not differ between $\mathrm{LG}$ and $\mathrm{OG}$ cohorts.

Large-scale, Asian-based RCTs that compared LDG with ODG showed no difference in the rate of mortality or morbidity between treatment cohorts [2-4], but wound problems, such as seroma and dehiscence, were reduced in the LDG arm of one study [4]. In addition, postoperative hospital stay was significantly shorter in LDG-based cohorts in two previous studies [3, 4]. However, only $4.9 \%$ of enrolled patients were ASA-PS class 3 in one of the RCTs, which excluded grade 4 or higher patients [4], and the other reports did not reveal the ASA-PS status. Previous studies using the NCD showed that $8.9-11.6 \%$ of patients underwent distal or total gastrectomy and were ASA-PS class $\geq 3[16,17,24$, 25]. The NCD-based studies showed LG to be associated with fewer wound problems and a shorter hospital stay than OG for both distal and total gastrectomy [24, 25]. Fewer wound problems and a shorter hospital stay were also found to be associated with LG in the present study, therefore, a laparoscopic approach may be beneficial, irrespective of ASA-PS class.

The ASA-PS classification system is a tool that not only evaluates the preoperative general condition for patients undergoing anesthesia, but also predicts postoperative morbidity and mortality in various types of operations. In the risk models for gastrectomy constructed from the NCD, ASA-PS class $\geq 3$ is an independent risk factor of operative mortality, both for distal gastrectomy [odds ratio (OR) 1.95, 95\% confidence interval (CI) 1.29-2.95] and total gastrectomy (OR 1.82, 95\% CI 1.37-2.42) [16, 17]. The 30-day mortality of distal and total gastrectomy was only $0.4 \%$ and $0.5 \%$ in previous NCD studies, respectively, including any patients of any ASA-PS class [24, 25]. However, the 30-day mortality was relatively high in this study. ASA-PS class $\geq 3$ is used clinically as a predictor of mortality after gastrectomy in the NCD risk calculator [28]. Moreover, ASA-PS class $\geq 3$ is also linked to postoperative complications after distal gastrectomy, such as cardiac events, events of the central nervous system, systemic sepsis, renal failure, and even surgical complication such as anastomotic leakage [15]. The current study showed high rates of pneumonia and sepsis, when compared to previous NCD studies examining distal and total gastrectomy (pneumonia, $1.6 \%$ and $2.5 \%$,sepsis, $0.2 \%$ and $0.6 \%$ ) [24, 25], while surgical complications in this study, such as leakage, pancreatic fistula, or abscess were not different from those of previous studies. In this study, few patients were ASA-PS class 4 and none were class 5 or 6 . Patients with ASA-PS class 4 have severe systemic disease that is poorly controlled or at end stage, and the population of these types of patients was small in another study of gastrectomy (4\%) [9]. Patients with ASA-PS class 5 or 6 commonly do not undergo distal or total gastrectomy because class 5 denotes a moribund patient who is not expected to survive without surgery and class 6 designates a patient who is declared brain dead and whose organs are being procured for donor purposes. Patients with extremely severe comorbidities might undergo alternative therapies, such as local resection or endoscopic resection, to prevent postoperative complications.

The current study has limitations worth noting. First, due to its nature as a retrospective observational study, the two groups retrieved for comparison between the two surgical approaches may have differed in terms of baseline characteristics or preoperative conditions. We tried to minimize the effect of potential confounding variables using propensity score matching, but the possibility of unobserved factors not considered could remain. Moreover, differences in surgeon and hospital characteristics 
were not taken into account and thus may have biased the estimated advantage of the LG approach. Since almost all operations performed in Japan are registered in the NCD, surgeons with varying levels of experience were present. Differences in both the LG- and OG-performing surgeons may influence the outcome of the two procedures. A similar problem exists at the hospital level, since different hospitals have varying perioperative care plans. The clinical pathway is commonly used for the perioperative management of gastrectomy, but earlier discharge might be planned for LG patients due to the earlier postoperative recovery of this surgery. We did not stratify the cohort by the treating institutions' volume, as the population of ASA-PS class $>3$ was small and modeling and matching in high/low volume centers separately would have led to unstable modeling. The reconstruction method may have an impact on individual postoperative complications, however, this information is not registered in the JSGS-NCD registry. The difference in reconstruction method between LG and OG was unknown in this study. However, an RCT study has shown that postoperative complications between Billroth-I and Roux-Y were not different [29]. The details or severity of comorbidities were unavailable, however, accuracy of the ASA-PS classification was secured by the audit system of the JSGS-NCD registry [22]. Details of mortality were also unavailable, which made it difficult to know the true reason for the differences in mortality between LG and OG patients. Second, we were unable to distinguish proximal from total gastrectomy, which prevented us from analyzing these types of surgery separately. Proximal gastrectomy might be performed for patients with poorer physical status instead of total gastrectomy, which is more invasive and is typically associated with more complications. These variables need to be further explored using the 2018 JSGS and newer data, where the two procedures are classified as different procedures. Total gastrectomy and distal gastrectomy were comprehensively analyzed in this study. To maximize the power of the analysis, we assumed that the risk ratio of LTG to OTG would not be different from the ratio of LDG-to-ODG, and designed the study based on the hypothesis described above. The incidence of individual postoperative complication is most likely different between total gastrectomy and distal gastrectomy.

However, it may be difficult to conduct a prospective study for patients with ASA-PS $\geq$ class 3 due to small patient populations, as well as the high rate of postoperative complication. Patients with severe comorbidities are sometimes not accepted in high-volume cancer centers with fewer expert physicians that treat these conditions. Therefore, a national database from both high-volume centers and general hospitals was useful for the present analysis of patients with a poor general condition.

\section{Conclusions}

Compared to OG, LG was associated with a decreased rate of in-hospital mortality and a lower incidence of several postoperative complications in patients with a poor physical status defined by ASA-PS class $\geq 3$. However, avoiding LG for such patients who can withstand OG may be unnecessary. LG may become a common treatment for gastric cancer, irrespective of ASA-PS class.

\section{Compliance with ethical standards}

Conflict of interest Hiraku Kumamaru and Hiroaki Miyata are affiliated with the Department of Healthcare Quality Assessment at the University of Tokyo. The department is a social collaboration department that is supported by grants from the National Clinical Database, Johnson \& Johnson K.K., and Nipro Co.

Ethical approval All procedures were conducted in accordance with the ethical standards of the respective national committees on human experimentation and with the Helsinki Declaration. The use of data from the registry for retrospective observational studies was approved by The Japanese Society of Gastroenterological Surgery committee and the Institutional Review Board of Tokyo Medical and Dental University committee. Individual written informed consent was waived because of the retrospective design.

\section{References}

1. Japanese Gastric Cancer Association. Japanese gastric cancer treatment guidelines 2014 (ver. 4). Gastric Cancer. 2017;20:1-19.

2. Katai H, Mizusawa J, Katayama H, Takagi M, Yoshikawa T, Fukagawa T, et al. Short-term surgical outcomes from a phase III study of laparoscopy-assisted versus open distal gastrectomy with nodal dissection for clinical stage IA/IB gastric cancer: Japan Clinical Oncology Group Study JCOG0912. Gastric Cancer. 2017;20:699-708.

3. Hu Y, Huang C, Sun Y, Su X, Cao H, Hu J, et al. Morbidity and mortality of laparoscopic versus open D2 distal gastrectomy for advanced gastric cancer: a randomized controlled trial. J Clin Oncol. 2016;34:1350-7.

4. Kim W, Kim HH, Han SU, Kim MC, Hyung WJ, Ryu SW, et al. Decreased morbidity of laparoscopic distal gastrectomy compared with open distal gastrectomy for stage I gastric cancer: shortterm outcomes from a multicenter randomized controlled trial (KLASS-01). Ann Surg. 2016;263:28-35.

5. Joris JL, Noirot DP, Legrand MJ, Jacquet NJ, Lamy ML. Hemodynamic changes during laparoscopic cholecystectomy. Anesth Analg. 1993;76:1067-71.

6. Critchley LA, Ho AM. Surgical emphysema as a cause of severe hypercapnia during laparoscopic surgery. Anaesth Intensive Care. 2010;38(6):1094-100.

7. Chang HM, Lee SW, Nomura E, Tanigawa N. Laparoscopic versus open gastrectomy for gastric cancer patients with COPD. J Surg Oncol. 2009;100:456-8.

8. Inokuchi M, Kojima K, Yamada H, Kato K, Enjoji M, Hayashi M, et al. Clinical outcomes of laparoscopy-assisted gastrectomy for 
patients with heart disease. Surg Laparosc Endosc Percutaneous Tech. 2013;23:69-73.

9. Inokuchi M, Kojima K, Kato K, Motoyama K, Sugita H, Sugihara K. Feasibility of laparoscopy-assisted gastrectomy for patients with poor physical status: a propensity-score matching study. Int J Surg. 2016;31:47-51.

10. Haynes SR, Lawler PG. An assessment of the consistency of ASA physical status classification allocation. Anaesthesia. 1995;50:195-9.

11. Sankar A, Johnson SR, Beattie WS, Tait G, Wijeysundera DN. Reliability of the American Society of Anesthesiologists physical status scale in clinical practice. Br J Anaesth. 2014;113:424-32.

12. Protopapa KL, Simpson JC, Smith NC, Moonesinghe SR. Development and validation of the surgical outcome risk tool (SORT). Br J Surg. 2014;101:1774-833.

13. Sauvanet A, Mariette C, Thomas P, Lozac'h P, Segol P, Tiret E, et al. Mortality and morbidity after resection for adenocarcinoma of the gastroesophageal junction: predictive factors. J Am Coll Surg. 2005;201:253-62.

14. Park JH, Kim DH, Kim BR, Kim YW. The American Society of Anesthesiologists score influences on postoperative complications and total hospital charges after laparoscopic colorectal cancer surgery. Medicine (Baltimore). 2018;97:e0653.

15. Kunisaki C, Miyata H, Konno H, Saze Z, Hirahara N, Kikuchi $\mathrm{H}$, et al. Modeling preoperative risk factors for potentially lethal morbidities using a nationwide Japanese web-based database of patients undergoing distal gastrectomy for gastric cancer. Gastric Cancer. 2017;20:496-507.

16. Kurita N, Miyata H, Gotoh M, Shimada M, Imura S, Kimura W, et al. Risk model for distal gastrectomy for distal gastrectomy when treating gastric cancer on the basis of data from 33,917 Japanese patients collected using a nationwide web-based data entry system. Ann Surg. 2015;262:295-303.

17. Watanabe M, Miyata H, Gotoh M, Baba H, Kimura W, Tomita $\mathrm{N}$, et al. Total gastrectomy risk model: data from 20,011 Japanese patients in a nationwide internet-based database. Ann Surg. 2014;260:1034-9.

18. Sankar A, Johnson SR, Beattie WS, Tait G, Wijeysundera DN. Reliability of the American Society of Anesthesiologists physical status scale in clinical practice. Br J Anesthsiol. 2014;113:424-32.

19. Suzuki H, Gotoh M, Sugihara K, Kitagawa Y, Kimura W, Kondo $\mathrm{S}$, et al. Nationwide survey and establishment of a clinical database for gastrointestinal surgery in Japan: targeting integration of a cancer registration system and improving the outcome of cancer treatment. Cancer Sci. 2011;102:226-30.

20. Gotoh M, Miyata H, Hashimoto H, Wakabayashi G, Konno H, Miyakawa S, et al. National Clinical Database feedback implementation for quality improvement of cancer treatment in Japan: from good to great through transparency. Surg Today. 2016;46:38-47.

21. Seto Y, Kakeji Y, Miyata T, Iwanaka T. National Clinical Database (NCD) in Japan for gastroenterological surgery: brief introduction. Ann Gastroenterol Surg. 2017;1:80-1.

22. Kanaji S, Takahashi A, Miyata H, Marubashhi S, Kakeji Y, Konno $\mathrm{H}$, et al. Initial verification of data from a clinical database of gastroenterological surgery in Japan. Surg Today. 2019;49:328-33.

23. Clavien PA, Barkun J, de Oliviera ML, Vauthey JN, Dindo D, Schulick RD, et al. The Clavien-Dindo classification of surgical complications: five-year experience. Ann Surg. 2009;250:187-96.

24. Kodera Y, Yoshida K, Kumamaru H, Kakeji Y, Hiki N, Etoh T, et al. Introducing laparoscopic total gastrectomy for gastric cancer in general practice: a retrospective cohort study based on a nationwide registry database in Japan. Gastric Cancer. 2018. https://doi. org/10.1007/s10120-018-0795-0.

25. Hiki N, Honda M, Etoh T, Yoshida K, Kodera Y, Kakeji Y, et al. Higher incidence of pancreatic fistula in laparoscopic gastrectomy. Real-world evidence from a nationwide prospective cohort study. Gastric Cancer. 2018;21:162-70.

26. Yang D, Dalton J. A unified approach to measuring the effect size between two groups using SAS. SAS Global Forum 2012 Paper $335,2012$.

27. Coca-Perraillon M. Local and global optimal propensity score matching. SAS Global Forum 2007 Paper 185, 2007.

28. National Clinical Database risk calculator (in Japanese). https:// www.ncd.or.jp/about/feedback.html. Accessed 16 June 2019.

29. Imamura H, Takiguchi S, Yamamoto K, Hirao M, Fujita J, Miyashiro I, et al. Morbidity and mortality results from a prospective randomized controlled trial comparing Billroth I and Roux-en-Y reconstructive procedures after distal gastrectomy for gastric cancer. World J Surg. 2012;36:632-7.

Publisher's Note Springer Nature remains neutral with regard to jurisdictional claims in published maps and institutional affiliations. 